\title{
Brain: Introduction
}

The Brain section of this book is the largest and covers a combination of normal variants, normal anatomy, and artifacts that can mimic disease. Although there is an attempt to organize such normal variations by region or anatomic structure, such a classification is inherently problematic because some normal variants can be in multiple locations (e.g., choroid plexus or dilated perivascular spaces), while some commonly encountered artifacts can occur anywhere in the brain (e.g., flow voids). Thus, this section of the book is generally organized starting from inferiorly at the skull base to more superiorly, while normal variants that can occur anywhere in the brain are generally placed in the middle. Also, artifacts or magnetic resonance related sequence phenomena that may simulate disease are placed toward the end of this section.

Some basic terminology and abbreviations regarding standard sequences is necessary, since magnetic resonance imaging (MRI) manufacturers unfortunately have not adopted one standard for sequences outside of the routine ones such as T1-weighted images, T2-weighted images, fluid-attenuated inversion recovery (FLAIR), and diffusion-weighted images. Thus, standard terminology used throughout this section and the remainder of the book is described below.

\section{$1.1 \quad$ Terminology}

1.5 T and $3 \mathrm{~T} 1.5$ Tesla and 3.0 Tesla MRI magnet field strengths
$3 \mathrm{D}$

BFFE

CECT

CISS

DWI

FFE

FLAIR

GE

GE T2*WI

IR

T1IR

T2IR

MiniP

MIP

MPR

MRA

TOF MRA

NECT

SWI

T1WI

T2WI

$\mathrm{T} 2 * \mathrm{WI}$

US
Three dimensional

Balance FFE (similar to CISS)

Contrast-enhanced computed tomography (CT)

Constructive interference in steady state, similar to T2WI, emphasizes cerebrospinal fluid hyperintensity

Diffusion-weighted image

Fast field echo (either T1- or T2-weighted)

Fluid-attenuated inversion recovery imaging

Gradient echo

Gradient echo T2*-weighted imaging

Inversion recovery imaging

T1-weighted IR imaging

T2-weighted IR imaging

Minimum intensity projection

Maximum intensity projection

Multiplanar reformat

MR angiography

Time-of-flight MRA

Non-enhanced CT

Susceptibility-weighted imaging

T1-weighted imaging

T2-weighted imaging

GE T2*WI

Ultrasound 